\title{
On the nonlinear viscoelastic behaviour of fresh and dried oil palm mesocarp fibres
}

\begin{abstract}
Investigation on viscoelastic behaviour of oil palm fibres was reported through experimental and finite element modelling study. The experimental work through tensile stress relaxation and cyclic tests revealed time-dependent behaviour and damage within the oil palm fibres. From the former test results, stresses of fresh fibres reduced more than the dried ones after 1 second relaxation, whereas increasing damage was observed under larger deformations from the latter test results. Finite element modelling results using Prony series viscoelastic model with damage function only agreed with small deformation test, whereas Parallel Rheological Framework viscoelastic model agreed with large deformation test. The former model can be used for biodegradation study, which does not involve large deformation, whereas the latter model is suitable for biocomposites study under large deformation.
\end{abstract}

Keyword: Oil palm fibres; Finite element analysis; Mechanical testing 